\title{
On Plurality Voting and Runoff Elections: Information Aggregation under Divided Majority*
}

\author{
Jacque $\mathrm{Gao}^{\dagger}$
}

July 15,2020

\begin{abstract}
This article compares Plurality Voting (PV) and two forms of Runoff Elections (RE) in a setting in which (i) there are two majority-preferred alternatives, (ii) a strong minority backs a third alternative which would make the majority strictly worse off, and (iii) some of the majority voters are uninformed about the "correct" majority alternative. I show that in the informative equilibrium in Majority Runoff Elections (MRE), uninformed majority voters vote randomly with strictly positive probability, achieving partial information aggregation, while they always abstain in Automatic Runoff Elections (ARE), achieving full information aggregation and strictly improving the majority's welfare. However, uninformed majority voters do not abstain in PV, resulting in less information aggregation than in both MRE and ARE.
\end{abstract}

Key Words: Epistemic Democracy; Information Aggregation; Voting

${ }^{*}$ I thank Mark Fey for valuable comments on the paper.

†Department of Political Science, University of Rochester. Email: ang.gao@ rochester.edu. 


\section{Introduction}

One of the main purposes of elections is to reach a better decision collectively than what any individual could have reached by herself through information aggregation. While information aggregation in Plurality Voting (PV) has been extensively studied (Feddersen and Pesendorfer 1996, 1999; Battaglini, Morton and Palfrey 2010), the study on how Runoff Elections (RE) aggregate information remains scant despite many informal conjectures (Duverger 1954; Riker 1982; Cox 1997). What's more, earlier works studying RE formally tend to leave important features out of the model (Piketty 2000; Martinelli 2002). More recently, Tsakas and Xefteris (2020) show that RE may aggregate information better in large societies. However, that still leaves open the question of how RE performs in small, finite sized societies. While in a spatial setup with sincere voting, Brusco et al. (2012) show that in Runoff Elections, only divergent equilibria, in which candidates choose distinct policy positions, are robust to free entry.

The mechanics of strategic voting in Runoff Elections are exactly what this article studies. Specifically, I analyze an election with a finite number of voters, in which (i) there are two majoritypreferred alternatives, (ii) a strong minority backs a third alternative which would make the majority strictly worse off, and (iii) some majority voters are uninformed about the "correct" majority alternative. Three forms of voting rules are compared: Plurality Voting (PV), Majority Runoff Elections (MRE) and Automatic Runoff Elections (ARE). In PV, the winner of the election is the

alternative receiving the most votes. In MRE, if an alternative obtains $50 \%$ of the votes or more in the first round, it wins the election outright. Otherwise, the two alternatives receiving the most votes in the first round will compete in a second round, while other alternatives get eliminated. In ARE, a second round is organized between the top two alternatives in the first round regardless of the number of votes each alternative gets.

Several results emerge. First, although informed majority voters always vote according to their signals in the informative equilibrium in both MRE and PV, uninformed majority voters abstain with strictly positive probability in MRE while they do not abstain in PV. The intuition behind the 
result is the following. In MRE, as long as the majority alternative passes the first round, it will win the election for sure in the second round against the Condorcet loser. Thus, uninformed voters have an incentive to let informed voters choose the alternative for them. However, abstention also risks letting the Condorcet loser steal the election by winning outright in the first round. As a result, uninformed voters will mix between voting randomly and abstention so that each one of them is indifferent between these two strategies. In PV, however, given that the minority is strong, i.e., the number of minority voters is greater than a half of the number of majority voters, an uninformed voter's vote can only be pivotal against the Condorcet loser. Thus, any empty ballot is just a vote wasted for the majority. As a result, uninformed voters always vote randomly in PV, impeding information aggregation. The result thus confirms previous conjectures that Runoff Elections are more conducive to information revelation than Plurality (Duverger 1954; Riker 1982; Cox 1997). It also conforms well to media perceptions of French presidential elections in particular, in which the only possibility for a Le Pen victory is to win the election outright in the first round.

Second, while Duverger's Law equilibria, in which only two candidates receive votes, survive sincere stability refinement (equilibria that are robust to the case in which voters may tremble by voting sincerely) in PV, such equilibria do not survive in MRE. Thus, the result improves upon Tsakas and Xefteris (2019), who show that MRE may admit Duvergerian equilibria in undominated strategies.

Third, the issue of a minority victory goes away if the 50\% threshold is eliminated in RE, in which case all uninformed voters will abstain in the first round and let informed voters choose the alternative for them. As a result, the "correct" majority alternative always wins the election as long as there is at least one informed majority voter, maximizing majority welfare. Thus, one policy implication of the analysis is to abolish the 50\% threshold in MRE and let the top two alternatives in the first round compete in a second round automatically.

Section 2 introduces the setup of the model. Section 3 analyzes key equilibrium properties of PV, MRE and ARE respectively, while Section 4 concludes. 


\section{Basic Setup}

I consider a voting environment in which there is an electorate of fixed and finite size who must elect an alternative $P$ out of three: $A, B$ and $C$.

There are two groups of voters, $n$ active majority voters and $m$ passive minority voters, and two states of nature: $\omega=\{a, b\}$. The prior probabilities of the two states of nature are the following: $\operatorname{Pr}(a)=\operatorname{Pr}(b)=\frac{1}{2}$, i.e., the two states of nature are equally likely to occur.

A majority voter's utility depends on the state of nature and the alternative being elected. Specifically, her utility is highest if the winning alternative matches the state of nature, intermediate if there is a mismatch between the two, and lowest if alternative $C$ is elected:

$$
\begin{aligned}
& U(A, a)=U(B, b)=1, \\
& U(A, b)=U(B, a)=0, \\
& U(C)=-1 .
\end{aligned}
$$

Minority voters, however, strictly prefer $C$ to either $A$ or $B$ and are indifferent between $A$ and $B$. Thus, it is a dominant strategy for them to vote for $C$ in any electoral system. I focus on the case in which the minority is strong, i.e., $\frac{n}{2}+1 \leq m \leq n-1$. Thus, although $C$ is a Condorcet loser, it is still possible for $C$ to win the election if majority voters split their votes between $A$ and $B$. For simplicity, I also assume that both $n$ and $m$ are even, so that $\frac{n}{2}+1<m<n-1$.

\subsection{Timing}

Before the election (time 0), nature chooses whether the state is in $a$ or in $b$. At time 1 , each voter receives a signal $s \in S \equiv\left\{s_{A}, s_{B}, s_{\psi}\right\}$ with the following conditional probabilities: 


$$
\begin{aligned}
& \operatorname{Pr}\left[s=s_{A} \mid \omega=a\right]=\operatorname{Pr}\left[s=s_{B} \mid \omega=b\right]=q, \\
& \operatorname{Pr}\left[s=s_{B} \mid \omega=a\right]=\operatorname{Pr}\left[s=s_{A} \mid \omega=b\right]=0, \\
& \operatorname{Pr}\left[s=s_{\psi} \mid \omega=a\right]=\operatorname{Pr}\left[s=s_{\psi} \mid \omega=b\right]=1-q,
\end{aligned}
$$

where $q \in(0,1)$. Thus, there are essentially two types of majority voters: informed voters and

uninformed voters. While informed voters know exactly the state of nature, uninformed voters only know the prior probabilities of the two states.

The election takes place at time 2, when the actual state of nature is still unobserved. Payoffs

realize at time 3, when the winner of the election and the actual state of nature are revealed, and each voter receives utility $U(P, \omega)$.

\subsection{Strategy Space and Equilibrium Concept}

In both PV and the first round of RE, each voter can vote for exactly one alternative or abstain, i.e.,

$$
\Sigma_{P V}=\Sigma_{R E 1}=\{A, B, C, \phi\}
$$

where $\phi$ denotes abstention and action $A$ (respectively, $B$ and $C$ ) denotes voting for $A$ (respectively, $B$ and $C$ ).

In PV, the winner of the election is the alternative receiving the most votes, with ties broken by fair dice. In MRE, if an alternative receives more than $50 \%$ of the votes in the first round, or if it receives exactly $50 \%$ of the votes in the first round while neither of the other two alternatives receives as many, this alternative wins the election outright in the first round. Otherwise, the two alternatives receiving the most votes in the first round will compete in a second round while the alternative that gets the smallest number of votes in the first round gets eliminated. Ties are again broken by fair dice, that is, if the second- and third-placed alternatives in the first round receive the same number of votes while the first-placed alternative does not obtain $50 \%$ of the votes or more, 
the second- and third-placed alternatives will have an equal probability of entering the second round. In ARE, a second round will be held between the top two alternatives in the first round regardless of the number of votes each alternative gets.

In the second round of RE, each voter can only choose among the two alternatives that qualify for the second round, and the alternative that gets the most votes in the second round becomes the winner of the election. Specifically, suppose there is a second round between $A$ and $C$, each voter's strategy space in the second round becomes

$$
\Sigma_{R E 2}=\{A, C, \phi\}
$$

A symmetric strategy is a mapping $\sigma: S \rightarrow \triangle\left(\Sigma_{R}\right), R \in\{P V, R E 1, R E 2\}$, where $\sigma_{s}(K)$ is the probability that the majority voter who receives signal s plays $K \in \Sigma_{R}$. The equilibrium concept used in this paper is Bayesian Nash equilibria (BNE) that satisfy sincere stability. Specifically, while uninformed voters do not tremble, informed voters may tremble by voting sincerely whenever it is possible $\left(\sigma_{s_{A}}(A)>\epsilon\right.$ whenever $A \in \Sigma_{R}$ and $\sigma_{s_{B}}(B)>\epsilon$ whenever $B \in \Sigma_{R}$, where $R \in\{P V, R E 1, R E 2\}$ and $\epsilon \rightarrow 0)$. Note that sincere stability refinement imposes that at least some pivot probabilities remain strictly positive, thus it automatically eliminates equilibria in weakly dominated strategies and is a stronger equilibrium concept than BNE with no weakly dominated strategies.

\section{Results}

\subsection{Plurality Voting}

This section analyzes key equilibrium properties of Plurality Voting, and Appendix Section A contains technical details and proofs. I show that in PV, both Duverger's Law equilibria, in which all majority voters concentrate their votes on a single majority alternative, and informative equilibria, in which informed majority voters vote according to their signals $\left(\sigma_{s_{A}}(A)=\sigma_{s_{B}}(B)=\right.$ 1), survive sincere stability refinement. 
To start, recall that in PV, the alternative that gets the most votes wins the election. Furthermore, given that $m>\frac{n}{2}$, the two majority alternatives, $A$ and $B$, can only be pivotal against the minority alternative, $C$, but not against each other. As a result, it is a weakly dominated strategy for any majority voter to abstain, as regardless of which majority alternative wins, the outcome is strictly better than letting the minority steal the election.

Lemma 1. In PV, abstention is a weakly dominated strategy for both informed and uninformed majority voters.

The next proposition shows that both Duverger's Law equilibria and informative equilibria survive sincere stability refinement in PV.

\section{Proposition 1. In Plurality Voting,}

1. there exist two Duverger's Law equilibria, i.e., $\sigma_{s}(A)=1$ for all $s \in\left\{s_{A}, s_{B}, s_{\psi}\right\}$ and $\sigma_{s}(B)=1$ for all $s \in\left\{s_{A}, s_{B}, s_{\psi}\right\}$

2. there exists an informative equilibrium such that $\sigma_{s_{A}}(A)=\sigma_{s_{B}}(B)=1$, and $\sigma_{s_{\psi}}(A)=$ $\sigma_{s_{\psi}}(B)=\frac{1}{2}$, i.e., informed voters vote sincerely, while uninformed voters do not abstain and vote randomly.

The above results reveal that PV is bad for the majority on two fronts. First, Duverger's Law equilibria, in which no information aggregation occurs, exist. The reasons behind this result are the following. Suppose we are in the equilibrium in which every majority voter votes for $A$. If the state is $a$, no tremble will happen. Thus, neither voting for $B$ nor abstention is a profitable deviation for either type of majority voters. If the state is $b$, informed voters will tremble by voting for $B$ with probability $\epsilon$. However, as $\epsilon$ gets closer to 0 , the probability that $B$ is pivotal against $C$ also gets smaller. Thus, a vote for $B$ looks increasingly like a wasted vote from the perspective of a majority voter. As a result, it is not optimal for any majority voter to deviate and vote for $B$.

Second, even in the informative equilibrium, uninformed voters still vote randomly with probability 1 in order to prevent a minority victory, which inevitably increases the probability of choosing the wrong majority alternative. More importantly, the attempt of uninformed voters cannot completely prevent a minority victory due to the large size of the minority. 
Thus, the previous analysis raises the following question: does there exist an electoral system such that it not only ensures a majority victory, but also does so by inducing uninformed voters to abstain, thus helping achieve full information aggregation?

\subsection{Majority Runoff Elections}

This section analyzes key equilibrium properties of Majority Runoff Elections, and Appendix Section B contains technical details and proofs. The next result shows that in MRE, Duverger's Law equilibria do not survive sincere stability refinement.

Proposition 2. In the first round of MRE, Duverger's Law equilibria do not survive sincere stability refinement, i.e., neither $\sigma_{s}(A)=1$ for all $s \in\left\{s_{A}, s_{B}, s_{\psi}\right\}$ nor $\sigma_{s}(B)=1$ for all $s \in\left\{s_{A}, s_{B}, s_{\psi}\right\}$ is an equilibrium.

The intuition behind the above result is the following. First, notice that since the only possibility for the Condorcet loser to win in MRE is to obtain greater than or equal to $50 \%$ of the votes in the first round, a split majority between $A$ and $B$ will not in itself lead to a minority victory. This is in stark contrast to PV, where a split majority alone may lead to a minority victory. Now, suppose every majority voter votes for $A$. For a single uninformed voter, it is clear that no pivotal event will occur if the state of nature is $a$, as everyone is already voting for $A$ and informed voters are not trembling. Thus, if her vote can make a difference, then it means the state of nature must be $b$. Knowing this and the fact that a split majority will not lead to a minority victory, this uninformed voter would rather deviate and vote for $B$.

The next proposition characterizes an informative equilibrium in MRE, such that informed voters vote sincerely, while uninformed voters mix among voting for $A$, voting for $B$ and abstention.

Proposition 3. In the first round of MRE, there exists an informative equilibrium such that $\sigma_{s_{A}}(A)=$ $\sigma_{s_{B}}(B)=1, \sigma_{s_{\psi}}(A)=\sigma_{s_{\psi}}(B)>0$ and $\sigma_{s_{\psi}}(\phi)>0$, i.e., informed voters vote sincerely, while uninformed voters mix between voting for $A$, voting for $B$ and abstention;

Thus, in the informative equilibrium in MRE, informed voters always vote according to their signals while uninformed voters abstain with strictly positive probability. As a result, information aggregation is relatively more efficient in the above equilibrium than in the informative equilibrium 
in PV. The intuition behind the result is straightforward. Notice that the key tradeoff for uninformed majority voters in the first round of MRE is between abstention, which increases both the likelihood of selecting the correct majority alternative and the likelihood of an outright minority victory in the first round, and voting randomly, which has the exact opposite effect. Thus, uninformed voters will mix between these two strategies in equilibrium so that each one of them is indifferent between voting randomly and abstention. This result is again consistent with the 2002 French presidential election, in which turnout in the first round was lower than in the second round. As the minority alternative, Jean-Marie Le Pen, passed the first round, all majority voters voted for the majority alternative, Jacques Chirac, in the second round, in order to ensure a majority victory.

Table 1 shows the abstention probability of uninformed voters under various specifications. As the table shows, uninformed voters are more likely to abstain when there are fewer minority voters ( $m$ is smaller), more majority voters ( $n$ is larger), and when the probability of being informed is larger ( $q$ is larger). In fact, the abstention probability can be quite high, reaching 0.77 when $q$ is equal to 0.9 and the number of uninformed and informed voters are 38 and 70 respectively. Thus, information can indeed aggregate quite efficiently in MRE.

Table 1: Abstention probabilities of uninformed voters

\begin{tabular}{||cccc||}
\hline $\mathrm{q}$ & $\mathrm{m}=32, \mathrm{n}=60$ & $\mathrm{~m}=38, \mathrm{n}=60$ & $\mathrm{~m}=38, \mathrm{n}=70$ \\
\hline \hline 0.5 & 0.34 & 0.22 & 0.35 \\
\hline 0.7 & 0.47 & 0.27 & 0.50 \\
\hline 0.9 & 0.73 & 0.71 & 0.77 \\
\hline
\end{tabular}

To conclude, MRE has two major advantages over PV. First, Duverger's Law equilibria, in which no information aggregates, do not exist in MRE. Second, in the informative equilibrium in MRE, uninformed voters abstain with strictly positive probability, which helps information aggregate more efficiently than in the informative equilibrium in PV. However, there is still room for improvements in MRE, as an outright minority victory in the first round is still possible. What's more, uninformed voters still do not abstain with probability 1 in the informative equilibrium in MRE, impeding information aggregation. The next section shows that one simple way to solve the problem is to eliminate the threshold requirement in the first round. 


\subsection{Automatic Runoff Elections}

Recall that in ARE, a second round will be held between the top two alternatives in the first round regardless of the number of votes each alternative gets. Thus, uninformed voters do not need to worry about the problem of an outright minority victory in the first round. As a result, they will completely entrust informed voters to pick the right alternative for them by abstaining in the first round with probability 1 .

Proposition 4. In the first round of ARE, there exists an unique informative equilibrium such that $\sigma_{s_{A}}(A)=\sigma_{s_{B}}(B)=1$ and $\sigma_{s_{\psi}}(\phi)=1$, i.e., informed voters vote sincerely while uninformed voters abstain.

The next result shows that majority voters are strictly better off in ARE than in both PV and MRE.

Proposition 5. Majority voters are strictly better off in the informative equilibrium in ARE than in the informative equilibrium in both $P V$ and MRE.

The majority are better off in ARE for two reasons. First, given that the second round will always be organized and the majority alternative will win for sure in the second round, it is impossible for the Condorcet loser to win the election in ARE. Second, given that uninformed voters always abstain in the first round, the correct majority alternative will be selected for sure as long as there is at least one informed voter! Thus, one policy prescription from the analysis is for countries with MRE to abolish the threshold requirement in first round.

\section{Conclusion}

Building on previous studies of multicandidate elections, this article compares how voters react to information differently in Plurality Voting and two forms of Runoff Elections. Three main results emerge. First, although informed majority voters always vote according to their signals in the informative equilibrium in both MRE and PV, uninformed majority voters abstain with strictly positive probability in MRE while they do not abstain in PV. Second, while Duverger's Law equilibria, in which only two candidates receive votes, survive sincere stability refinement in 
PV, such equilibria do not survive in either MRE or ARE. Third, the issue of a minority victory goes away if the $50 \%$ threshold requirement is eliminated in RE, and the "correct" majority alternative always wins the election as long as there is at least one informed majority voter. Thus, abolishing the $50 \%$ threshold of MRE may be desirable in enhancing the majority's welfare.

\section{References}

Battaglini, Marco, Rebecca B Morton and Thomas R Palfrey. 2010. “The Swing Voter's Curse in the Laboratory." Review of Economic Studies 77(1):61-89.

Brusco, Sandro, Marcin Dziubiński and Jaideep Roy. 2012. “The Hotelling-Downs Model With Runoff Voting." Games and Economic Behavior 74(2):447-469.

Cox, Gary W. 1997. Making Votes Count: Strategic Coordination in the World's Electoral Systems. Cambridge, UK: Cambridge University Press.

Duverger, Maurice. 1954. Political Parties. New York: Wiley.

Feddersen, Timothy J and Wolfgang Pesendorfer. 1996. “The Swing Voter's Curse." American Economic Review pp. 408-424.

Feddersen, Timothy J and Wolfgang Pesendorfer. 1999. "Abstention in Elections with Asymmetric Information and Diverse Preferences." American Political Science Review 93(02):381-398.

Martinelli, Cesar. 2002. "Simple Plurality versus Plurality Runoff with Privately Informed Voters." Social Choice and Welfare 19(4):901-919.

Piketty, Thomas. 2000. "Voting as Communicating." Review of Economic Studies 67(1):169-191.

Riker, William H. 1982. “The Two-Party System and Duverger's Law: An Essay on the History of Political Science.” American Political Science Review 76(4):753-766.

Tsakas, Nikolas and Dimitrios Xefteris. 2019. "Stress-Testing the Runoff Rule in the Laboratory." SSRN Working Paper . 
Tsakas, Nikolas and Dimitrios Xefteris. 2020. "Information Aggregation with Runoff Voting." SSRN Working Paper. 